\title{
Article
}

\section{Dielectric constants of bulk ferroelectric PZTmeasured by terahertz time-domain spectroscopy}

Naftaly, M, Cain, M, Lepadatu, Serban, Buchacher, T and Allam, J

Available at http://clok.uclan.ac.uk/16468/

Naftaly, M, Cain, M, Lepadatu, Serban ORCID: 0000-0001-6221-9727, Buchacher, $T$ and Allam, J (2016) Dielectric constants of bulk ferroelectric PZTmeasured by terahertz time-domain spectroscopy. Advances in Applied Ceramics: Structural, Functional and Bioceramics Journal - Advances in Psychiatric Treatment . pp. 260-263. ISSN 1743-6753

It is advisable to refer to the publisher's version if you intend to cite from the work. /10.1080/17436753.2015.1130199

For more information about UCLan's research in this area go to http://www.uclan.ac.uk/researchgroups/ and search for <name of research Group>.

For information about Research generally at UCLan please go to http://www.uclan.ac.uk/research/

All outputs in CLoK are protected by Intellectual Property Rights law, including Copyright law. Copyright, IPR and Moral Rights for the works on this site are retained by the individual authors and/or other copyright owners. Terms and conditions for use of this material are defined in the policies page.

\section{CLoK}

Central Lancashire online Knowledge www.clok.uclan.ac.uk

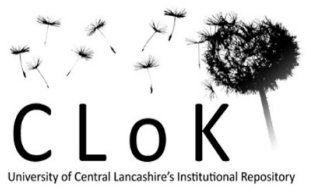




\title{
Dielectric constants of bulk ferroelectric PZT measured by THz time-domain spectroscopy
}

Mira Naftaly ${ }^{1}$, Markys G Cain ${ }^{1}$, Serban Lepadatu ${ }^{1,3}$, Till Buchacher ${ }^{1,2}$ and Jeremy Allam ${ }^{1}$

1. National Physical Laboratory, Hampton Road, Teddington, Middlesex TW11 0LW, UK

2. Advanced Technology Institute, University of Surrey, Guildford, Surrey, GU2 7XH, UK

3. University of Central Lancashire, Jeremiah Horrocks Institute, Preston, PR1 2HE, UK

\begin{abstract}
The complex permittivity of bulk ceramic ferroelectric of nominal composition $\mathrm{PbZr}_{0.4} \mathrm{Ti}_{0.6} \mathrm{O}_{3}$ was measured in the range $0.2-2 \mathrm{THz}$ using transmission time-domain spectroscopy. The results indicate strong absorption and dispersion in this frequency range often seen in highly disordered and polar materials. The results are compared to equivalent thin film data in the literature, and significant differences in the real and imaginary permittivity suggest that substrate clamping and degree of polarisation of the ferroelectric thin film materials affect dielectric properties even at these high frequencies.
\end{abstract}

\section{Introduction}

Wireless communication technologies are continuously expanding to ever-higher frequencies. Experimental wireless links have already been demonstrated at frequencies up to $300 \mathrm{GHz}$ [1]. These technological developments will demand advances in dielectric and other functional materials for passive and possibly active devices, whose design will require accurate knowledge of dielectric properties at sub- $\mathrm{THz}$ and $\mathrm{THz}$ frequencies.

Ferroelectric and piezoelectric materials underpin a wide range of existing technologies, such as sensors, actuators and transducers, capacitors, pyroelectric detectors, medical ultrasound, and ferroelectric memory. Ultra-fast processes in ferroelectrics are also of direct technological interest for non-linear optics, photonics, telecommunications and optical data storage [2]-[4].

Despite their industrial relevance, it is surprising that very little literature has been published on the dielectric properties of ferroelectric and piezoelectric materials at $\mathrm{THz}$ frequencies, with most of the 
high frequency work focused on mm wavelength (microwave) applications [5]-[7]. The great majority of the published data has been derived indirectly from $\mathrm{THz}$ reflectivity measurements [8]-[16], where the calculations involved are subject to large uncertainties [17]. This is especially relevant in materials with high reflectivity, where both real and imaginary dielectric constants have large values, as is the case in the industry standard piezoelectric family of ceramics known as PZT (i.e. $\mathrm{Pb}\left(\mathrm{Zr}_{\mathrm{x}}, \mathrm{Ti}_{1-\mathrm{x}}\right) \mathrm{O}_{3}-$ perovskite solid solutions). Moreover, most of the cited studies were performed using Fourier Transform Spectroscopy (FTS), a technique that offers ultra-broadband measurement over as much as 30-3000 $\mathrm{cm}^{-1}(1-100 \mathrm{THz})$. However, the method does not provide a direct measurement of the phase, which therefore makes derivations of optical constants from reflectivity data even more problematic [17], since they must be calculated from the Kramers-Kronig dispersion integrals Chapter 9 in [18].

THz time-domain spectroscopy (TDS) offers a much narrower range of frequencies than FTS, typically 0.1-4 THz, but has the important advantage of yielding a direct measurement of both amplitude and phase shift, making possible a much more accurate determination of the real and imaginary dielectric constants. Chen et al. [7] used reflection THz TDS to measure the refractive index of epitaxial PbZro.52Tio.48 $\mathrm{O}_{3}$ film on $\mathrm{SrTiO}_{3}$ substrate at $1 \mathrm{THz}$ as a function of applied electric field. $\mathrm{Li}$ et al. [6] used the goniometric form of reflection THz TDS to obtain the dielectric constants of a $\mathrm{PbZr} 0.4 \mathrm{Ti}_{0.6} \mathrm{O}_{3}$ film on Si substrate at discrete frequencies in the 0.6-1.4 THz range. However, because these studies used reflectivity measurements, the values of the derived optical constants are subject to quite large uncertainties associated with this approach.

In contrast, transmission time-domain spectroscopy provides a direct measurement of the complex dielectric constant with comparatively low uncertainties. Kwak et al. [5] used transmission THz TDS to measure the real and imaginary dielectric constants of sol-gel grown $\mathrm{PbZr} 0.52 \mathrm{Ti} 0.48 \mathrm{O}_{3}$ and $\mathrm{PbZr} 0.3 \mathrm{Ti}_{0.7} \mathrm{O}_{3}$ films in the $0.1-2 \mathrm{THz}$ range, demonstrating the advantages of the technique for these materials. Similarly, Buixaderas et al. [19], [20] included transmission THz TDS in the range 0.150.6 THz in their comprehensive study of dielectric properties of PLZT ( $\mathrm{Pb}, \mathrm{La}, \mathrm{Zr}, \mathrm{Ti})$ materials at 
frequencies between $10^{2}-10^{14} \mathrm{~Hz}$ and at temperatures of $20-800 \mathrm{~K}$, as well as in their similar study of PZT materials [21].

With so little data published on the dielectric properties of bulk materials, we present in this paper transmission THz TDS measurements on bulk ceramic $\mathrm{PbZr}_{0.4} \mathrm{Ti}_{0.6} \mathrm{O}_{3}$ in the range 0.2-2 $\mathrm{THz}$. The dielectric constants are compared with those measured on thin films grown by epitaxial and sol-gel processes.

\section{Experimental techniques}

Bulk sintered ceramic plates of $\mathrm{Pb}\left(\mathrm{Zr}_{1-\mathrm{x}} \mathrm{Ti}_{\mathrm{x}}\right) \mathrm{O}_{3}$ with nominally tetragonal structure were sourced from a commercial supplier of PZT ceramics. The material composition was measured using energy-dispersive $\mathrm{X}$-ray spectroscopy and confirmed to be Ti-rich, with $\mathrm{x} \sim 0.6$. At $\mathrm{THz}$ frequencies, PZT has a high refractive index combined with high absorption. In consequence, and in order for transmission measurements to be made possible, the PZT samples must be mechanically thinned to less than about $50 \mu \mathrm{m}$. In these experiments, three samples were produced by grinding down and polishing the bulk ceramic to thicknesses between 20-30 $\mu \mathrm{m}$. Film thickness was measured using the gauge block technique, with an uncertainty of $\pm 1 \mu \mathrm{m}$ [22]. Since these films were too fragile to handle or mount, they were sandwiched between two plates of polymethylpentene (TPX® from Mitsui Chemicals) each 2 mm thick. TPX® was chosen because of its high THz transparency [23] combined with optical transparency and good mechanical stiffness. Two similar plates of TPX® contacted together served for reference measurements.

The $\mathrm{THz}$ time-domain spectrometer [24] had a commonly employed configuration with four $90^{\circ}$ parabolic mirrors. The pump laser was a mode-locked Ti:Sapphire laser (Femtosource) with a $20 \mathrm{fs}$ pulse length and a centre wavelength of $800 \mathrm{~nm}$, producing an average power of $450 \mathrm{~mW}$. The $\mathrm{THz}$ source was a photoconductive emitter fabricated on semi-insulating GaAs biased to $200 \mathrm{~V}$ with unipolar square-wave modulation at $10 \mathrm{kHz}$. The $\mathrm{THz}$ signal was detected by monitoring the electro-optic birefringence of a $0.5 \mathrm{~mm}$ thick $\mathrm{ZnTe}$ crystal using a pair of balanced photodiodes. The dynamic range 
of the system was approximately 2000 at $1 \mathrm{THz}$. For the measurement, the PZT sample was placed approximately $5 \mathrm{~mm}$ away from the focal plane of $\mathrm{THz}$ beam, in order to minimize wavefront distortion which would be caused by placing the samples at the exact focus [25].

\section{Experimental Results}

The refractive indices $(n)$ and absorption coefficients $(\alpha)$ of PZT samples were calculated from the frequency-domain transmission data obtained by Fast Fourier Transform of the THz transients, using the equations [19]:

$$
\begin{gathered}
n(\omega)=1+\frac{\left(\phi_{\text {ref }}-\phi_{\text {sample }}\right) c}{\omega d} \\
T(\omega)=1-\frac{\left(n-n_{S}\right)^{2}+k^{2}}{\left(n+n_{S}\right)^{2}+k^{2}} \\
k(\omega)=\frac{\alpha c}{2 \omega} \\
\alpha(\omega)=-\frac{2}{d} \ln \left(T \frac{E_{\text {sample }}}{E_{\text {ref }}}\right)
\end{gathered}
$$

where $\phi$ and $E$ are respectively the phase and amplitude of the THz signal, $\omega$ is its frequency, and $c$ is the speed of light in vacuum; $k$ is the extinction coefficient. The sample thickness is $d$, and the refractive index of TPX plates is $n_{s}=1.45$ [6]. The transmission factor $T$ accounts for losses arising from Fresnel reflections. Note that when $k$ is non-negligible, as it is for PZT, Eqs. 2-4 must be solved iteratively.

Fig. 1 shows the obtained refractive index and absorption coefficient of the PZT samples, and values at selected frequencies are listed in Table 1. Three different samples were measured, with thicknesses of 20, 24 and $29 \mu \mathrm{m}$. Each sample was measured at 3 different locations, with 4 scans at each location. The results from all three samples are similar within the measurement uncertainty, indicating consistency of the material properties and the absence of inhomogeneous surface effects arising from sample preparation (e.g. scattering, surface contamination). 


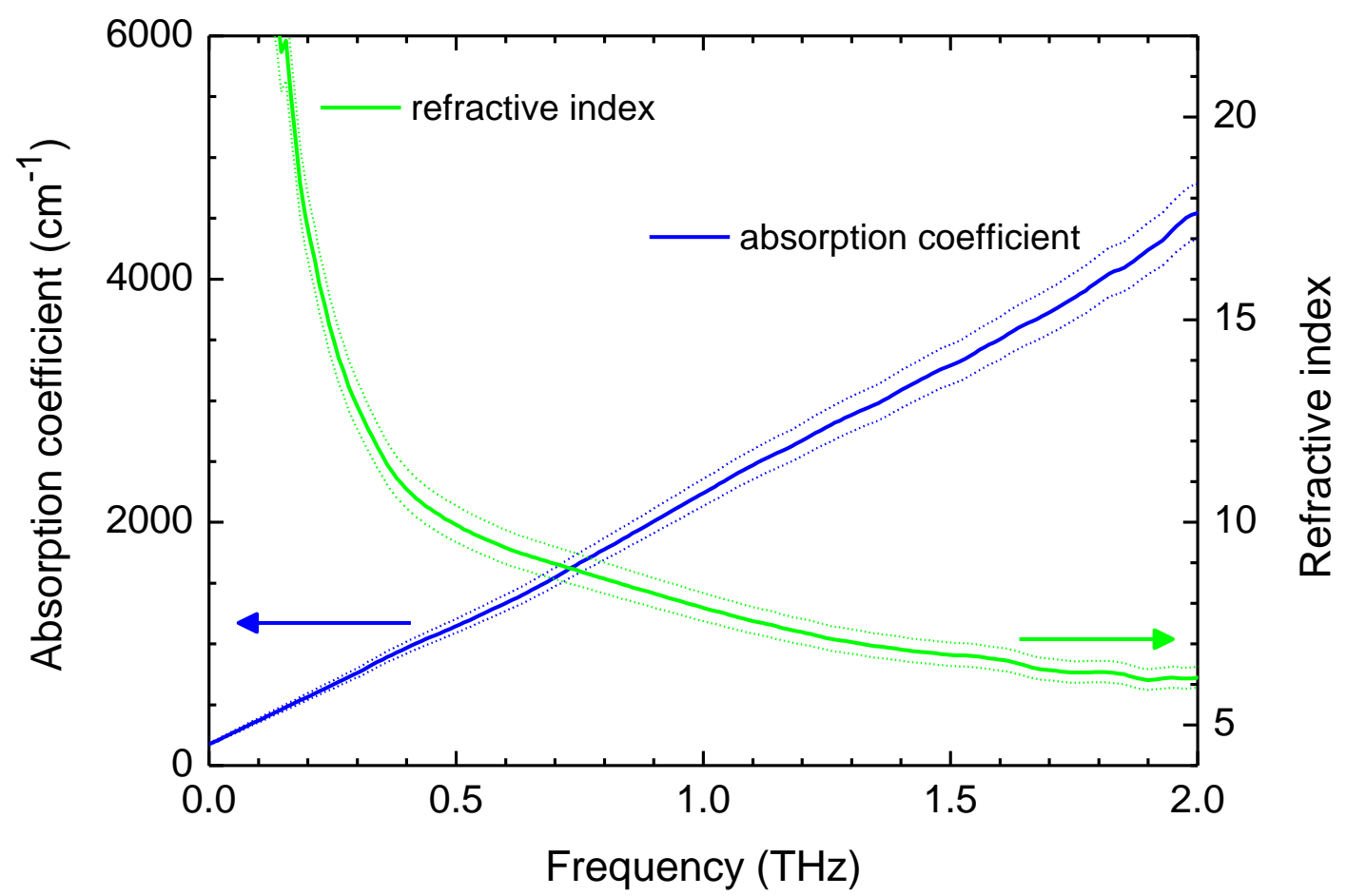

Fig. 1. Refractive index and absorption coefficient of PZT calculated from the transmission data according to Eqs. 1-4. The data represent measurements on 3 samples whose thicknesses were 20, 24 \& $29 \mu \mathrm{m}$ (with an uncertainty of $\pm 1 \mu \mathrm{m}$ ). The dashed lines represent measurement uncertainty arising from uncertainty in the sample thickness, which accounts for the main contribution to the error in this measurement.

Table 1. Refractive indices, absorption coefficients and real and imaginary permittivity of $\mathrm{PbZr}_{0.4} \mathrm{Ti}_{0.6} \mathrm{O}_{3}$ at $0.25,0.5,1.0$ and $1.5 \mathrm{THz}$.

\begin{tabular}{|l|l|l|l|l|}
\hline Frequency (THz) & 0.25 & 0.5 & 1.0 & 1.5 \\
\hline Refractive index & $14.6 \pm 1.4$ & $9.9 \pm 0.5$ & $7.9 \pm 0.4$ & $6.7 \pm 0.3$ \\
\hline Absorption coefficient $\left(\mathrm{cm}^{-1}\right)$ & $660 \pm 30$ & $1150 \pm 60$ & $2250 \pm 120$ & $3290 \pm 160$ \\
\hline$\varepsilon ’$ & $171 \pm 9$ & $69 \pm 3$ & $34 \pm 1$ & $18 \pm 1$ \\
\hline$\varepsilon^{\prime \prime}$ & $184 \pm 9$ & $108 \pm 5$ & $84 \pm 4$ & $70 \pm 3$ \\
\hline
\end{tabular}


The refractive index has large dispersion, falling from $\sim 20$ at $0.2 \mathrm{THz}$ to $\sim 8$ at $2 \mathrm{THz}$. The absorption coefficient is very large, and increases steeply from $\sim 500 \mathrm{~cm}^{-1}$ at $0.2 \mathrm{THz}$ to $\sim 7000 \mathrm{~cm}^{-1}$ at $2 \mathrm{THz}$. Both these features are indicative of relaxations on $\mathrm{THz}$ timescales [26]. The complex permittivity was calculated from the absorption and refractive index [21] according to:

$$
\varepsilon=\varepsilon^{\prime}+i \varepsilon^{\prime \prime}=(n+i k)^{2}=\left(n^{2}+k^{2}\right)+i(2 n k)
$$

and is shown in Fig. 2.

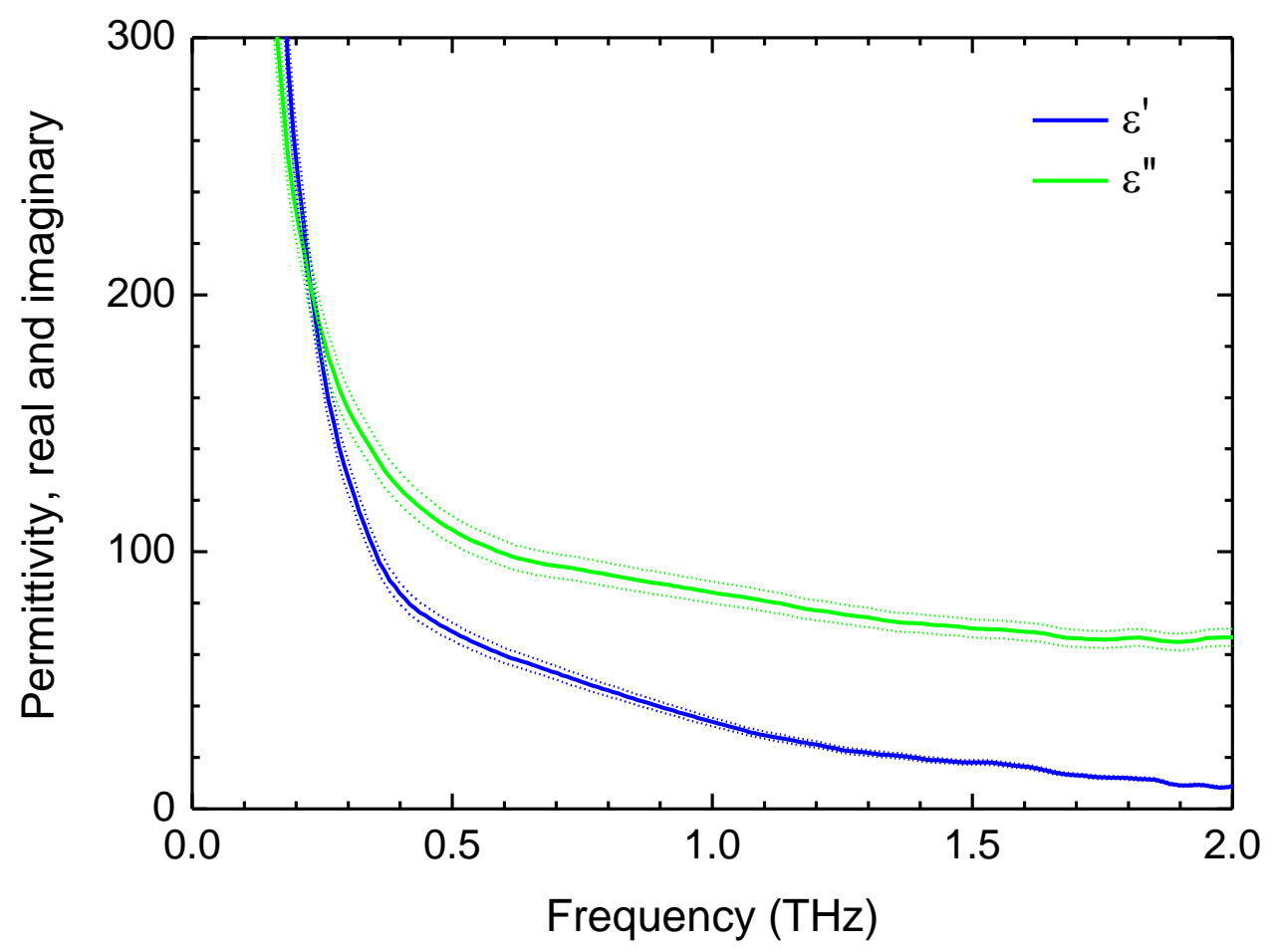

Fig. 2. The calculated permittivity of PZT - dashed lines represent measurement uncertainty. Note the $\mathrm{y}$-axis is the same for real and imaginary permittivity at these frequencies.

\section{Discussion}

From our data and calculated values of the real and imaginary values of permittivity we conclude the following: 
1. Our polished lamella of PZT exhibit an increase in permittivity with decreasing frequency similar to that observed in the literature. The absolute values differ by a factor of about 2 , with our data being approximately half the values reported for similar compositions in the work of Buixaderas, for example [21].

2. Buixaderas et al used TDS to measure PZT ceramics (polished to a similar thickness as our samples in this report) and also noted the increase in permittivity from a high frequency value of approximately 150 at $1 \mathrm{THz}$ to approx. 200 at their lowest measured frequency $\left(6 \mathrm{~cm}^{-1}\right.$ or about $0.2 \mathrm{THz}$ ) at room temperature, and for a similar composition [21]. Lower temperature measurements exhibited a flat permittivity, with all measured (calculated) imaginary permittivity passing through a peak before declining at low frequencies. The imaginary permittivity obtained in this work do not show a maximum or peak in response which is observed by Buixaderas and (weakly indicated) by Kwak [5], although an increase in imaginary permittivity with decreasing frequency is characteristic of these materials generally. Our data extends down to approximately $0.2 \mathrm{THz}\left(\sim 6 \mathrm{~cm}^{-1}\right)$, and the peaks reported in [21] occur around this frequency or below, so it is not surprising that this feature was not observed in our study. The discussion in Buixaderas explained the huge peak in imaginary permittivity at low frequency as an indicator of a $\mathrm{THz}$ mode contributing significantly to the high permittivity observed in their range of PZT compositions (which is approx. our composition), indicating that the main softening towards the morphotropic phase boundary takes place in this lower frequency range. The permittivity data presented in the literature shows great sensitivity to the chemical composition of the PZT ceramic - especially around the morphotropic phase boundary - and because the exact composition of commercially sourced materials is a closely guarded secret, a direct comparison of the absolute values of permittivity to other published work is not considered scientifically robust.

3. Kwak et al. [5] measured thin films of $\mathrm{Pb}\left(\mathrm{Zr}_{1-\mathrm{x}} \mathrm{Ti}_{\mathrm{x}}\right) \mathrm{O}_{3}$ with $\mathrm{x}=0.3$ and $\mathrm{x}=0.52$ grown on $\mathrm{MgO}$ single crystal substrates, also using time domain spectroscopy in the range $0.2-2 \mathrm{THz}$. For both compositions they obtain values of $\varepsilon^{\prime}$ and $\varepsilon^{\prime \prime}$ at $1 \mathrm{THz}$ roughly 3 times larger than our results. Apart from the different compositions, the most important difference is the use of a 
substrate-supported highly textured (oriented) thin PZT film, whilst the material studied in this paper was bulk (polycrystalline) PZT. Significant differences in the real and imaginary permittivity suggest that substrate clamping and degree of polarisation (induced through textured film growth) of the ferroelectric material affect dielectric properties even at these high frequencies. The effect of the substrate on the calculation of $\varepsilon^{\prime}$ and $\varepsilon^{\prime \prime}$ has been considered, and it is well known that ferroelectric thin films exhibit much lower polarisation at lower frequencies due to the clamping effect of the substrate at the interface [27], [28]. The clamped dielectric displacement vector due to interfacial strain also results in a modified dielectric polarization compared to bulk, though the changes in permittivity would depend critically on preferred orientation, strain, thickness, depolarising layers, boundary effects and more [2], [29]. The exact effect of the interfacial strain on the electronic and ionic polarisabilities is still under debate, however it does emphasize the need for measurements on bulk samples as well as thin film samples.

4. The THz TDS system used in these measurements was calibrated for frequency and linearity of response [17]. In preparing the samples, particular attention was given to obtaining uniform thickness and near-optical surface finish, and the enclosing TPX plates were likewise tested, thus reducing uncertainties arising from sample quality [17]. The optical constants were calculated from the data by applying Eqs. 1-4 iteratively (unlike, for example in Kwak [5]) to obtain highly accurate parameter values. The PZT material, as mentioned above, is intrinsically highly variable, with its permittivity strongly dependent on the chemical composition and the fabrication process. The great variability of published results emphasises the need for accurate measurements of $\mathrm{THz}$ permittivity on a range of compositions and types of material grown under different fabrication conditions.

\section{Conclusions}

The dielectric permittivity of an industrially sourced tetragonal (Ti-rich) PZT ceramic has been determined at $\mathrm{THz}$ frequencies by transmission time-domain spectroscopy. The measurement uncertainties are reduced with this method compared to others found in the literature, such as reflection 
Fourier transform spectroscopy. Both the real and imaginary permittivity differ from reported measurements on bulk ceramic and thin films of similar material compositions. The nature of the $\mathrm{THz}$ mode (soft mode) explored by Buixaderas was not observed in our data, most likely because of our composition being far from the MPB and the characteristic peak in their imaginary permittivity data being below our measurement frequency band. The strong dependence of THz permittivity on detailed characteristics of chemical composition, microstructure, manufacturing methods, and form of material (bulk or thin film) demonstrates the need for the accurate measurement of reference and commercial bulk materials, as presented here.

\section{Acknowledgements}

This work was funded by the National Measurement Office of the UK Department of Business Innovation and Skills. 


\section{References}

[1] T. Nagatsuma, S. Horiguchi, Y. Minamikata, Y. Yoshimizu, S. Hisatake, S. Kuwano, N. Yoshimoto, J. Terada, and H. Takahashi, "Terahertz wireless communications based on photonics technologies," Opt. Express, vol. 21, no. 20, pp. 23736-12, 2013.

[2] J. F. Scott, "Applications of Modern Ferroelectrics," Science, vol. 315, no. 5814, pp. 954 959, Feb. 2007.

[3] J. F. Scott, "Prospects for Ferroelectrics: 2012-2022," ISRN Materials Science, vol. 2013, no. 7, pp. 1-24, 2013.

[4] M. Fiebig and R. V. Pisarev, "Nonlinear optics - a powerful tool for the investigation of magnetic structures," Journal of Magnetism and Magnetic Materials, vol. 272, pp. E1607E1611, May 2004.

[5] M. H. Kwak, S. B. Kang, K.-C. Kim, S. Y. Jeong, S. Kim, B. H. Yoo, D. C. Chung, H. C. Ryu, D. S. Jun, M. C. Paek, and K. Y. Kang, "Dielectric Characteristics of Pb(Zr, Ti)O 3Films on MgO Single Crystal Substrate by Terahertz Time Domain Spectroscopy," Ferroelectrics, vol. 422, no. 1, pp. 19-22, Jan. 2011.

[6] M. Li, J. Fortin, J. Y. Kim, G. Fox, and F. Chu, "Dielectric constant measurement of thin films using goniometric terahertz time-domain spectroscopy," Selected Topics in ..., 2001.

[7] L. Chen, Y. Zhang, Q. Guo, D. Zhang, X. Zhong, and J. Yuan, "Terahertz electro-optic properties of PbZr0.52Ti0.48O3 and BaTiO3 ferroelectric thin films," Appl. Phys. Lett., vol. 105, no. 11, pp. 112903-5, Sep. 2014.

[8] V. Sivasubramanian, V. Murthy, B. Viswanathan, and M. Sieskind, "Morphotropic phase transition studies in PZT by far-infrared reflectance spectroscopy," J. Phys.: Condens. Matter, vol. 8, no. 14, p. 2447, 1996.

[9] V. Zelezny, P. Simon, F. Gervais, and T. Kala, "Soft mode behavior in PZT compounds by infrared reflectivity spectroscopy," Materials Research Bulletin, vol. 22, no. 12, pp. 1695$1702,1987$.

[10] Y. M. Poplavko and V. G. Tsykalov, Permittivity of Barium Titanate at Millimeter Wavelengths, vol. 8, no. 10. Soviet Physics Solid State, 1967, pp. 2490-2491.

[11] O. Kersten and G. Schmidt, "Dielectric dispersion in PZT ceramics," Ferroelectrics, vol. 67, no. 1, pp. 191-197, 1986.

[12] I. Fedorov, J. Petzelt, and V. Zelezny, "Far-infrared dielectric response of PbTiO3 and PbZr1-xTixO3 thin ferroelectric films," Journal of Physics: ..., 1995.

[13] C. A. Guarany, L. Pelaio, and E. B. Araújo, "Infrared studies of the monoclinic-tetragonal phase transition in $\mathrm{Pb}$ (Zr, Ti) O3 ceramics," Journal of Physics: ..., 2003.

[14] E. Buixaderas, D. Nuzhnyy, P. Vaněk, I. Gregora, J. Petzelt, V. Porokhonskyy, L. Jin, and D. Damjanovic, "Lattice dynamics and dielectric response of undoped, soft and hard $\mathrm{PbZr}$ 0.42Ti 0.58O 3," Phase Transitions, vol. 83, no. 10, pp. 917-930, Oct. 2010.

[15] E. B. Araújo, K. Yukimitu, and J. Moraes, "Monoclinic-tetragonal phase transition in $\mathrm{Pb}$ (Zr1-xTix) O3 studied by infrared spectroscopy," Journal of Physics: ..., 2002.

[16] E. B. Araújo, C. A. Guarany, K. Yukimitu, J. C. S. MORAES, and J. A. Eiras, "Structural Phase Transitions of PbZr 0.52Ti 0.48O 3Ceramic: An Infrared Spectroscopy Study," Ferroelectrics, vol. 337, no. 1, pp. 145-151, Aug. 2006.

[17] M. Naftaly, Terahertz Metrology. Artech House., 2015.

[18] M. Bass and O. S. O. America, Handbook of Optics: Fundamentals, techniques, and design, no. 1. McGraw-Hill, 1995.

[19] E. Buixaderas, D. Nuzhnyy, S. Veljko, S. Kamba, M. Savinov, J. Petzelt, and M. Kosec, "Far-infrared and dielectric spectroscopy of relaxor ferroelectric $(\mathrm{Pb}[\mathrm{sub} 1-\mathrm{x}] \mathrm{La}[\mathrm{sub}$ x])(Zr[sub 0.4]Ti[sub 0.6])O[sub 3]," J. Appl. Phys., vol. 101, no. 7, pp. 074106-9, 2007.

[20] E. Buixaderas, V. Bovtun, S. Veljko, M. Savinov, P. Kužel, I. Gregora, S. Kamba, and I. Reaney, "Ultrabroadband dielectric spectroscopy and phonons in $(\mathrm{Pb}[\mathrm{sub} 1-\mathrm{x} / 2] \mathrm{La}[\mathrm{sub}$ x])(Zr[sub 0.9]Ti[sub 0.1])O[sub 3]," J. Appl. Phys., vol. 108, no. 10, pp. 104101-11, 2010.

[21] E. Buixaderas, D. Nuzhnyy, J. Petzelt, L. Jin, and D. Damjanovic, "Polar lattice vibrations and phase transition dynamics in $\mathrm{Pb}\left(\mathrm{Zr}_{-}\{1-\mathrm{x}\} \mathrm{Ti}\{\{\mathrm{x}\}) \mathrm{O}_{-}\{3\}\right.$," Phys. Rev. B, vol. 84, no. 18, 
p. 184302, Nov. 2011.

[22] D. Flack, "CMM Verification: Good Practice Guide No.40,” NPL, Teddington, 40, Jul. 2001.

[23] A. Podzorov and G. Gallot, "Low-loss polymers for terahertz applications," Appl. Opt., vol. 47, no. 18, pp. 3254-3257, Jun. 2008.

[24] W. Withayachumnankul, B. M. Fischer, H. Lin, and D. Abbott, "Uncertainty in terahertz time-domain spectroscopy measurement," J. Opt. Soc. Am. B, vol. 25, no. 6, pp. 1059-1072, Jun. 2008.

[25] S. Ahmed, J. Savolainen, and P. Hamm, "The effect of the Gouy phase in optical-pumpTHz-probe spectroscopy," Opt. Express, vol. 22, no. 4, pp. 4256-11, 2014.

[26] S. Kasap and P. Capper, R.W. Whatmore, Ferroelectric Materials pp597-623 in Springer Handbook of Electronic and Photonic Materials. Springer, 2006.

[27] K. Lefki and G. Dormans, "Measurement of piezoelectric coefficients of ferroelectric thin films," J. Appl. Phys., vol. 76, no. 3, pp. 1764-1767, 1994.

[28] M. Stewart, S. Lepadatu, L. N. McCartney, M. G. Cain, L. Wright, J. Crain, D. M. Newns, and G. J. Martyna, "Electrode size and boundary condition independent measurement of the effective piezoelectric coefficient of thin films," APL Mater., vol. 3, no. 2, pp. 026103-8, Feb. 2015.

[29] A. V. Kimmel, J. Íñiguez, M. G. Cain, and P. V. Sushko, "Neutral and Charged Oxygen Vacancies Induce Two-Dimensional Electron Gas Near SiO 2/BaTiO 3Interfaces," J. Phys. Chem. Lett., vol. 4, no. 2, pp. 333-337, Jan. 2013. 